Research Paper

\title{
Preoperative AFU Is a Useful Serological Prognostic Predictor for Colorectal Liver Oligometastasis Patients Undergoing Hepatic Resection
}

\author{
Yuxiang Deng ${ }^{*}$, Yujie Zhao ${ }^{1 *}$, Wenhua Fan ${ }^{1 *}$, Jianhong Peng ${ }^{1}$, Xiao Luo ${ }^{2}$, Yiwen $\mathrm{Mo}^{3}$, Binyi Xiao1, Lin \\ Zhang ${ }^{\bowtie}$, and Zhizhong Pan ${ }^{\bowtie}$
}

1. Department of Colorectal Surgery, State Key Laboratory of Oncology in South China, Collaborative Innovation Center for Cancer Medicine, Sun Yat-sen University Cancer Center, Guangzhou, China.

2. Department of Ultrasound, State Key Laboratory of Oncology in South China, Collaborative Innovation Center for Cancer Medicine, Sun Yat-sen University Cancer Center, Guangzhou, China.

3. Department of Nuclear Medicine, State Key Laboratory of Oncology in South China, Collaborative Innovation Center for Cancer Medicine, Sun Yat-sen University Cancer Center, Guangzhou, China.

4. Department of Clinical Laboratory, State Key Laboratory of Oncology in South China, Collaborative Innovation Center for Cancer Medicine, Sun Yat-sen University Cancer Center, Guangzhou, China.

* These authors contributed equally to this work

$\triangle$ Corresponding authors: Zhizhong Pan: Phone: +86-20-87343124; Fax: +86-20-87343609; E-mail: panzhzh@sysucc.org.cn; Lin Zhang: Phone: +86-20-87343438; Fax: +86-20-87343199; E-mail: zhanglin@sysucc.org.cn

(C) The author(s). This is an open access article distributed under the terms of the Creative Commons Attribution License (https://creativecommons.org/licenses/by/4.0/). See http://ivyspring.com/terms for full terms and conditions.

Received: 2018.11.15; Accepted: 2019.08.05; Published: 2019.08.28

\begin{abstract}
Background: Preoperative alpha-l-fucosidase (AFU) has been used as a diagnostic biomarker for several cancers, but its role as a prognostic predictor in colorectal cancer liver oligometastasis (CLOM) patients after radical surgery has not been well defined. This study aimed to investigate the prognostic significance of preoperative serum AFU for CLOM patients after hepatic resection.

Methods: A retrospective data set was collected to evaluate the prognostic value of preoperative AFU in CLOM patients after radical hepatic resection. A total of 269 patients with histopathologically confirmed CLOM were enrolled. The optimal cut-off value of preoperative AFU was determined using $X$-tile software. Univariate and multivariate analyses were used to identify the prognostic significance of preoperative serum AFU.

Results: The X-tile software showed that the optimal cut-off value of preoperative AFU was set at 30.8 U/L. Patients with preoperative AFU $\leq 30.8$ and $>30.8$ were classified into high and low AFU groups, respectively. Female patients and those with a single liver metastasis had a higher tendency to have a preoperative AFU $\leq 30.8 \mathrm{U} / \mathrm{L}$; patients with lower clinical risk score (CRS) were more likely to have AFU $>30.8 \mathrm{U} / \mathrm{L}$ than patients with higher CRS. The results showed that preoperative AFU was an independent prognostic factor for overall survival (OS) $(P=0.041)$. Patients with a preoperative AFU $\leq 30.8 \mathrm{U} / \mathrm{L}$ had a lower OS rate than those with AFU>30.8 U/L. Furthermore, for patients with lower CRS scores (0-2), the tendency clearly showed that patients with higher preoperative AFU had a better prognosis $(\mathrm{P}=0.029)$.

Conclusions: Higher preoperative serum AFU can predict better survival in CLOM patients after hepatic resection, especially for CLOM patients with lower CRS scores.
\end{abstract}

Key words: preoperative AFU, CLOM, prognosis, OS

\section{Introduction}

The global burden of cancer worldwide using the GLOBOCAN 2018 estimated that colorectal cancer
(CRC) was the fourth most common cancer in incidence and the second leading cause of cancer 
death [1]. The liver is the most frequent site of metastatic disease. For patients with colorectal cancer liver metastasis (CRLM), hepatic resection is the main curative choice [2]. In the latest European Society for Medical Oncology (ESMO) guidelines, there are two categories for metastatic CRC: oligometastatic disease (OMD) and widespread systemic disease [3]. OMD is a state of metastatic disease that is limited in total disease burden, according to the limited amount of clinical or radiographic evidence [4, 5]. OMD represents a disease state that exists in a transitional zone between localized and widespread systemic diseases. It has a genuine potential for cure when patients receive complete $\mathrm{R} 0$ resection of their metastases [6, 7]. Because the prognoses of these two types of patients are quite different, the treatment strategies are also different. To date, few studies have highlighted the clinical survival of colorectal liver oligometastases (CLOM) patients who undergo curative resection. Therefore, identifying efficient prognostic factors for CLOM patients is urgently needed to screen for high-risk subgroups and to develop adequate therapeutic interventions for maximum therapeutic effectiveness.

a-L-fucosidases (AFU), a lysosomal enzyme in nature[8], is widely expressed in many types of cells. AFU catalyses the hydrolytic cleavage of terminal fucose residues, but its physiological functions are not completely understood. Previous observations indicated that human AFU is downregulated in highly aggressive human tumours, such as neuroblastomas [9], breast cancer [10], and colorectal cancers [11]. Serum and salivary AFU activity were significantly higher in OPC and oral cancer patients compared to controls [12]. Since elevated fucose levels are preferentially expressed in metastatic foci versus primary tumours [13-15], it has been suggested that the study of altered fucose in tumour cells could be useful in searching new treatment targets [16]. AFU has also been used as a biomarker for the early diagnosis of hepatocellular carcinoma (HCC) [17], as well as for its potential usefulness in clinical diagnosis [18-21]. As for its prognostic value, preoperative AFU is also a powerful prognostic indicator for HCC [22].

For CRC, previous study had revealed the prognostic predicting role of serum AFU activity in non-metastatic CRC patients [23]. However, to our knowledge, no studies have focused on the predictive role of preoperative AFU as an effective indicator of the prognosis of CLOM patients undergoing curative hepatic resection. In addition, the optimal cut-off value of AFU for the prediction of oncologic outcomes remains controversial. In this study, we aimed to identify the optimal AFU cut-off value and to investigate the prognostic impact of preoperative
AFU in patients with CLOM who underwent curative hepatic resection.

\section{Materials and Methods}

\section{Patient population}

We retrospectively examined data from 425 colorectal cancer liver metastasis patients who underwent curative resection at Sun Yat-sen University Cancer Center from January 2005 to December 2016. The enrolled patients met the following inclusion criteria: (1) histologically confirmed adenocarcinoma; (2) colorectal liver oligometastases ( $\leq 5$ metastases); (3) no preoperative extrahepatic metastases; and (4) R0 resection both for primary lesions and metastases. We excluded 156 patients based on the following exclusion criteria: number of liver metastases $>5(n=45)$; preoperative extrahepatic metastases $(n=40)$; $R 1$ or $R 2$ resection $(n=$ $25)$; loss to follow-up within 3 months $(n=1)$; incomplete pathological data $(\mathrm{n}=37)$; and incomplete AFU data $(n=8)$. The final cohort thus consisted of 269 patients (Figure 1). This study was conducted with the approval of the Institute Research Ethics Committee of the Sun Yat-Sen University Cancer Center.

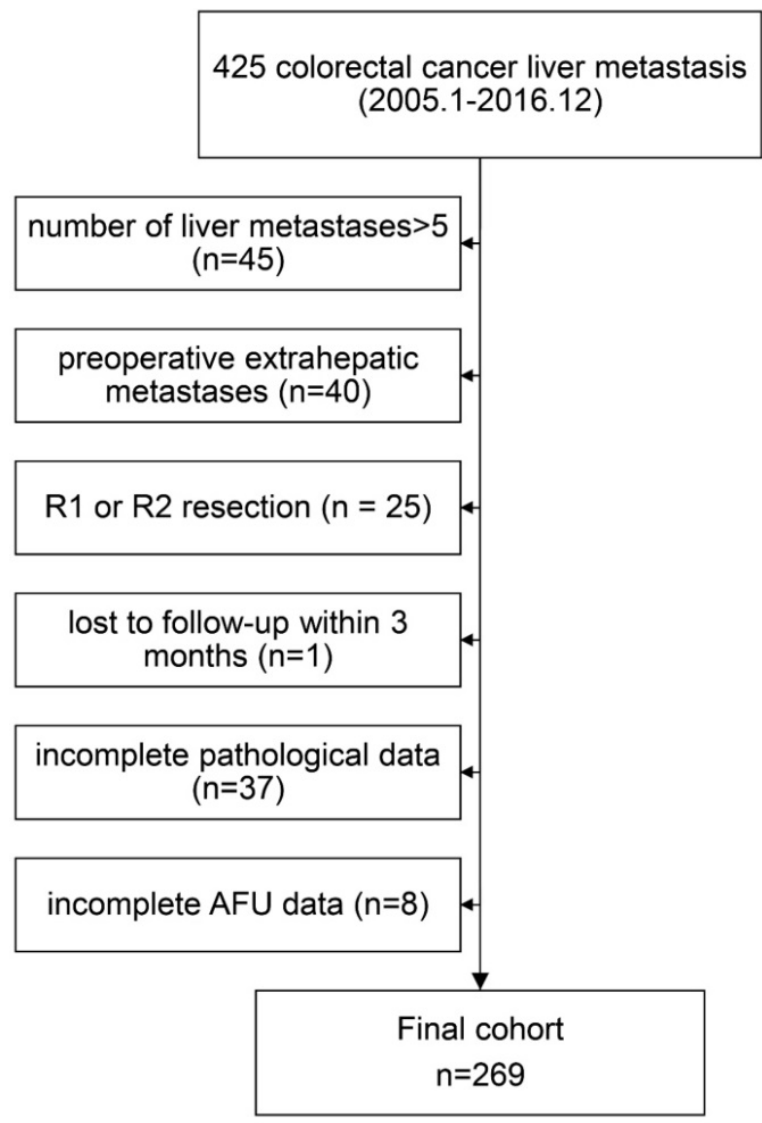

Figure 1. Flow chart of the analysed patients. 


\section{Clinical risk score (CRS)}

The clinical risk score (CRS) was used to predict recurrence after hepatic resection for metastatic CRC patients, which was also used as a stratifying factor in our study. The following five clinical criteria were chosen as the criteria for CRS: nodal status of primary, disease-free interval from the primary to discovery of liver metastases of $<12$ months, number of tumours $>1$, preoperative carcinoembryonic antigen (CEA) level $>200 \mathrm{ng} / \mathrm{ml}$, and size of the largest tumour $>5$ $\mathrm{cm}$. Each criterion was assigned one point according to the total score of CRS. Patients were divided into two groups: a low-risk group (score: 0-2) and a high-risk group (score: 3-5) [24].

\section{Patient treatments}

Primary tumours were staged according to the seventh edition of the UICC-TNM staging system for colorectal cancer. Preoperative tumour status was evaluated using a combination of colonoscopy, computed tomography (CT), ultrasonography, magnetic resonance imaging (MRI), and positron emission tomography. The decision and timing of surgical resection of the primary tumour was based on clinical assessment by a surgical oncologist in conjunction with a multidisciplinary team. The extent and nature of hepatic surgery were similarly individualized for each patient based on rigorous clinicopathological evaluation.

\section{Serum biological factors}

Patients received routine serum tests for biochemical detection between 7 and 10 a.m. within 7 days before the hepatic operation, and samples were centrifuged at $3500 \mathrm{~g} / \mathrm{min}$ for $8 \mathrm{~min}$ to allow serum separation. The levels of AFU were measured in serum using a Hitachi 7600 automatic biochemical analyser (Hitachi High-Technologies, Tokyo, Japan). CEA and cancer antigen 19-9 (CA19-9) were detected using an electrochemiluminescence immunoassay system (Elecsys 1601; Roche, Basel, Switzerland) according to the manufacturer's instructions, and the cut-off values for CEA and CA19-9 were $5 \mathrm{ng} / \mathrm{mL}$ and $37 \mathrm{U} / \mathrm{mL}$, respectively.

\section{Follow-up}

The follow-up protocol included evaluations every 3 months for the first 2 years after surgery, every 6 months for the third to fifth years and once every year thereafter. Evaluations at each visit included obtaining a complete blood count, evaluations of CEA and CA199 levels, and a physical examination. Chest radiography, abdominal and pelvic CT, colonoscopy, and pelvic MRI were conducted every year. Overall survival was defined as time from liver resection to death or last follow-up. Patients alive at the last follow-up date were regarded as randomly censored. Follow-up was terminated in September 2018.

\section{Statistical analysis}

The cut-off value for AFU was assessed using X-tile 3.6.1 software (Yale University, New Haven, $\mathrm{CT}$, USA), identified from the minimum $\mathrm{P}$ value according to OS. Statistical analyses were performed using SPSS 20.0 software (IBM, Chicago, IL, USA) and GraphPad Prism 7 software (GraphPad Software, Inc., San Diego, CA, USA). We compared categorical variables using the chi-square $\left(\chi^{2}\right)$ test. The KaplanMeier method was used to estimate the survival rates for the different groups, and differences in survival were compared with the log-rank test. A multivariate Cox proportional hazards analysis was performed using variables with $\mathrm{P}$ values less than 0.05 in univariate analysis. $\quad \mathrm{P}<0.05$ was considered statistically significant.

\section{Results}

\section{Patient characteristics and optimal cut-off value of AFU}

Table 1 shows the patients' clinical and pathological characteristics. The mean age of all patients was $56 \pm 12$ years, and $66.2 \%$ of the patients were male. X-tile software was used to determine 30.8 as the optimal cut-off value for AFU (Figure 2). Based on the serum AFU level, 189 patients were classified into the low-AFU (AFU 30.8 ) group, and 80 patients were classified into the high-AFU (AFU>30.8) group. The associations between AFU level and various clinicopathological features are shown in Table 2. Among all 269 patients, there were no significant differences in the distribution of age, tumour location or TN stage between the two groups. Moreover, there were no significant differences in the histological grade, liver metastases tumour size, hepatic resection timing, or CEA level and CA199 level of the patients in the low- and high-AFU groups. Females, patients who had single liver metastasis and those with higher CRS were more frequently observed in the low-AFU group ( $\mathrm{P}=0.046 ; \mathrm{P}=0.041$; and $\mathrm{P}=0.007$, respectively).

\section{Association between AFU and survival}

The median follow-up time for all patients was 36.7 months (range 2.3-151.7 months) after liver resection. During that period of time, 98 (36.4\%) patients were ultimately dead because of tumour progression. An obvious difference was observed in patients with different levels of preoperative AFU. Kaplan-Meier analysis indicated that the 3-year OS rate in the low-AFU group was significantly lower 
than that in the high-AFU group $(67.5 \%$ vs $81.3 \%$, $\mathrm{P}=0.041$; Figure $3 \mathrm{~A}$ ).

Table 1. Clinicopathologic features of patients involved in this study.

\begin{tabular}{|c|c|c|}
\hline Characteristics & & No. $(\%)$ \\
\hline \multirow[t]{2}{*}{ Age (years) } & $\leq 60$ & $159(59.1)$ \\
\hline & $>60$ & $110(39.1)$ \\
\hline \multirow[t]{2}{*}{ Gender } & Female & $91(33.8)$ \\
\hline & Male & $178(66.2)$ \\
\hline \multirow[t]{2}{*}{ Primary tumor site } & Colon & $172(63.9)$ \\
\hline & Rectum & $97(36.1)$ \\
\hline \multirow[t]{4}{*}{ T stage } & 1 & $1(0.4)$ \\
\hline & 2 & $25(9.3)$ \\
\hline & 3 & $148(55.0)$ \\
\hline & 4 & $95(35.3)$ \\
\hline \multirow[t]{3}{*}{ N stage } & 0 & $115(42.8)$ \\
\hline & 1 & $97(36.1)$ \\
\hline & 2 & $57(21.2)$ \\
\hline \multirow[t]{2}{*}{ Histological grade } & Well/moderate & $237(88.1)$ \\
\hline & Poor & $32(11.9)$ \\
\hline \multirow{2}{*}{$\begin{array}{l}\text { Liver metastases tumor size } \\
(\mathrm{cm})\end{array}$} & $\leq 2.3$ & $136(50.6)$ \\
\hline & $>2.3$ & $133(49.4)$ \\
\hline \multirow[t]{2}{*}{ Liver metastases number } & Single & $140(52.0)$ \\
\hline & Multiple & $129(48.0)$ \\
\hline \multirow[t]{2}{*}{ Hepatic resection timing } & Synchronous & $161(59.9)$ \\
\hline & Metachronous & $108(40.1)$ \\
\hline \multirow[t]{2}{*}{ CEA (ng/ml) } & $\leq 5$ & $117(43.5)$ \\
\hline & $>5$ & $152(56.5)$ \\
\hline \multirow[t]{2}{*}{ CA199 (U/ml) } & $\leq 37$ & $205(76.2)$ \\
\hline & $>37$ & $64(23.8)$ \\
\hline \multirow[t]{2}{*}{ CRS } & $0-2$ & $186(69.1)$ \\
\hline & $3-5$ & $83(30.9)$ \\
\hline \multirow[t]{2}{*}{ AFU (U/L) } & $\leq 30.8$ & $189(70.3)$ \\
\hline & $>30.8$ & $80(29.7)$ \\
\hline
\end{tabular}

Abbreviations: CEA, carcinoembryonic antigen; CA19-9, cancer antigen 19-9; CRS, clinical risk score; AFU, alpha-l-fucosidase.

\section{Univariate and multivariate analyses of prognostic factors}

The univariate and multivariate analyses are summarized in Table 3. The univariate analyses showed that OS was significantly associated with primary tumour site $(\mathrm{P}=0.003), \mathrm{N}$ stage $(\mathrm{P}=0.002)$, number of liver metastases $(\mathrm{P}=0.011)$ and $\mathrm{AFU}$ $(\mathrm{P}=0.043)$. In addition, Cox multivariate analysis showed that the following were independent poor prognostic factors for OS: primary tumour in rectum $(\mathrm{P}<0.001)$, advanced $\mathrm{N}$ stage $(\mathrm{P}<0.001)$, multiple liver metastases $(P=0.001)$, and operative AFU level $\leq 30.8$ $(\mathrm{P}=0.001)$.

\section{Prognostic analysis in patients with different AFU levels in accordance with CRS}

In our study, CLOM patients were divided into two subgroups according to CRS. One hundred and eighty-six $(69.1 \%)$ patients belonged to the CRS-low group (score: $0-2)$, and $83(30.9 \%)$ patients were in the CRS-high group (score: $3-5$ ). We conducted a survival analysis based on the CRS group, and an interesting result was found. For patients with low CRS, a significant deterioration in survival was observed for patients with low AFU compared with patients with high AFU (3-year OS: $85.9 \%$ vs $73.0 \%, \mathrm{P}=0.029$; Figure 3B). However, for patients with a higher CRS (score: 3-5), despite the tendency towards survival diversity, there was no significant difference in the 3-year OS rate between high AFU and low AFU ( $\mathrm{P}=0.143)$ (Figure 3C).
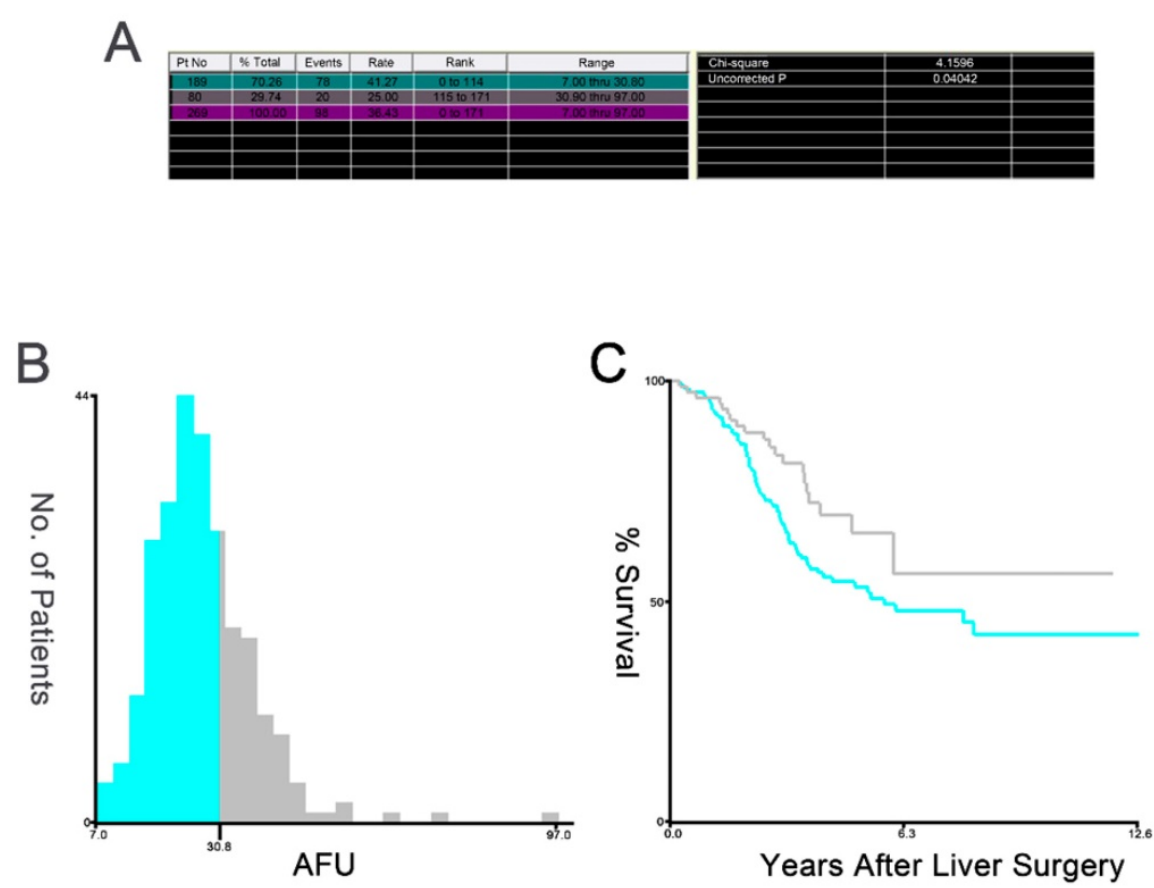

Figure 2. X-tile plots of the AFU and the OS of patients with CLOM who underwent curative resection. X-tile plots showing X2 values with cut-off points to generate the lowand high-AFU subgroups. (A) The optimal cut-off value of the AFU was 30.8 at the maximum X2 value of 4.1596 . (B) Histogram of the entire cohort divided into low-AFU and high-AFU subgroups according to the optimal cut-off value of 30.8. Blue bars represent the low-AFU group, and grey bars represent the high-AFU group. (C) Kaplan-Meier plot of OS in groups stratified using the optimal cut-off value of AFU. Blue curves represent the low-AFU group, and grey curves represent the high-AFU group. 

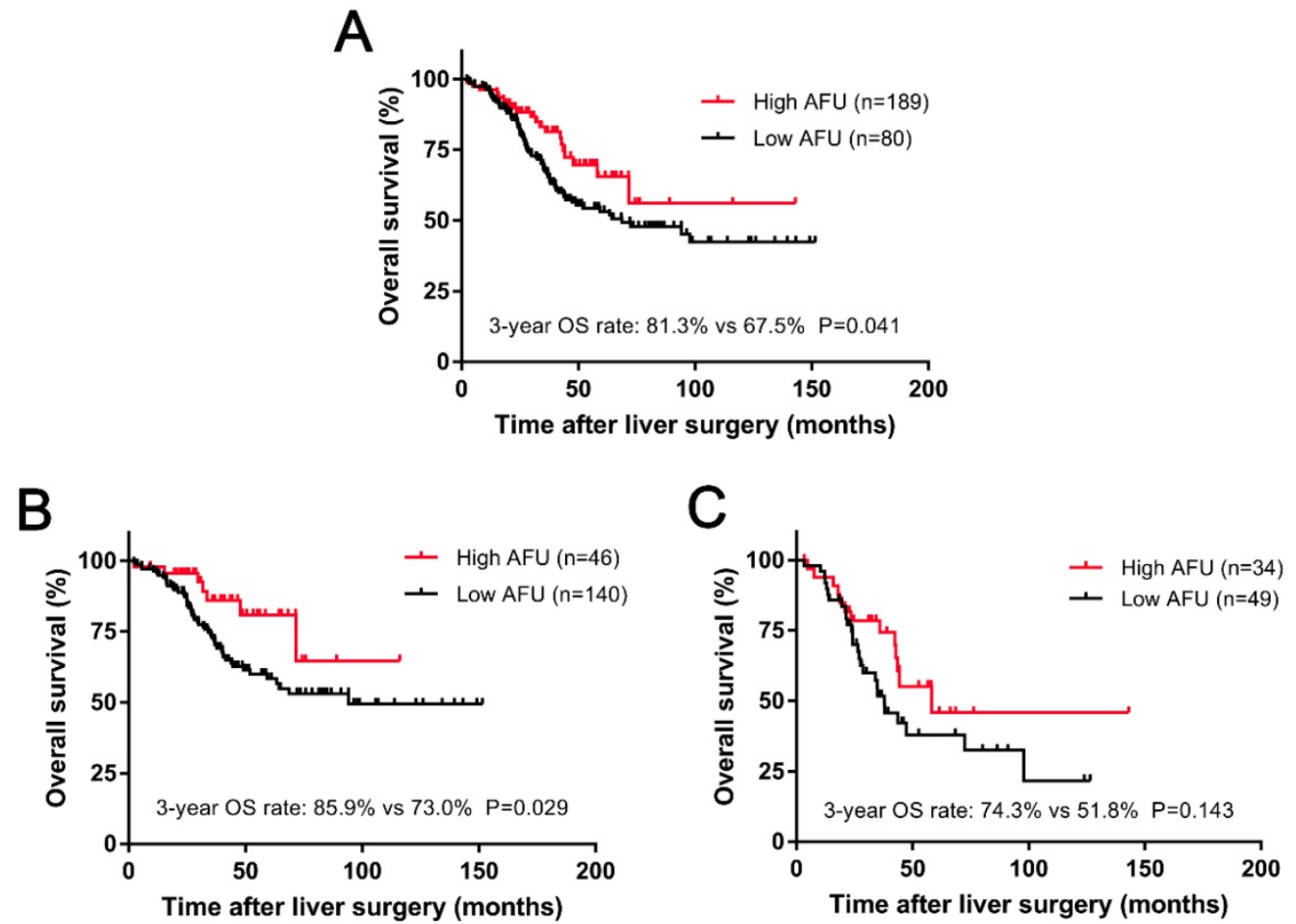

Figure 3. Kaplan-Meier curves for 3-year OS (A) based on the AFU. Kaplan-Meier curves for 3-year OS (B) based on the AFU in subgroups with low CRS (score: 0-2) and for 3-year OS (C) based on the AFU in subgroups with high CRS (score: 3-5).

Table 2. Relationships between AFU and patient characteristics

\begin{tabular}{|c|c|c|c|}
\hline \multirow[b]{2}{*}{ Characteristics } & \multicolumn{3}{|l|}{ AFU (U/L) (n=269) } \\
\hline & $\leq 30.8(\mathrm{n}=189), \mathrm{n}(\%)$ & $>30.8(\mathrm{n}=80), \mathrm{n}(\%)$ & P value \\
\hline \multicolumn{4}{|l|}{ Age (years) } \\
\hline$\leq 60$ & $105(55.6)$ & $54(67.5)$ & 0.069 \\
\hline$>60$ & $84(44.4)$ & $26(32.5)$ & \\
\hline \multicolumn{4}{|l|}{ Sex } \\
\hline Female & $71(37.6)$ & $20(25.0)$ & 0.046 \\
\hline Male & $118(62.4)$ & $60(75.0)$ & \\
\hline \multicolumn{4}{|c|}{ Primary tumor site } \\
\hline Colon & $123(65.1)$ & 49 (61.2) & 0.550 \\
\hline Rectum & $66(34.9)$ & $31(38.8)$ & \\
\hline \multicolumn{4}{|l|}{ T stage } \\
\hline $1-3$ & $125(66.1)$ & 49 (61.2) & 0.443 \\
\hline 4 & $64(33.9)$ & $31(38.8)$ & \\
\hline \multicolumn{4}{|l|}{ N stage } \\
\hline 0 & $85(45.0)$ & $30(37.5)$ & 0.257 \\
\hline $1-2$ & $104(55.0)$ & $50(62.5)$ & \\
\hline \multicolumn{4}{|c|}{ Histological grade } \\
\hline Well/moderate & $165(87.3)$ & $72(90.0)$ & 0.532 \\
\hline Poor & $24(12.7)$ & $8(10.0)$ & \\
\hline \multicolumn{4}{|c|}{ Liver metastases tumor size $(\mathrm{cm})$} \\
\hline$\leq 2.3$ & $98(51.9)$ & $38(47.5)$ & 0.514 \\
\hline$>2.3$ & $91(48.1)$ & $42(52.5)$ & \\
\hline \multicolumn{4}{|c|}{ Liver metastases number } \\
\hline Single & $106(56.1)$ & $34(42.5)$ & 0.041 \\
\hline Multiple & $83(43.9)$ & $46(57.5)$ & \\
\hline \multicolumn{4}{|c|}{ Hepatic resection timing } \\
\hline Synchronous & $118(62.4)$ & $43(53.8)$ & 0.184 \\
\hline Metachronous & $71(37.6)$ & $37(46.2)$ & \\
\hline \multicolumn{4}{|l|}{ CEA (ng/ml) } \\
\hline$\leq 5$ & $84(44.4)$ & $33(41.2)$ & 0.629 \\
\hline$>5$ & $105(55.6)$ & $47(58.8)$ & \\
\hline \multicolumn{4}{|l|}{ CA199 (U/ml) } \\
\hline$\leq 37$ & $149(78.8)$ & $56(70.0)$ & 0.120 \\
\hline$>37$ & $40(21.2)$ & $24(30.0)$ & \\
\hline \multicolumn{4}{|l|}{ CRS } \\
\hline $0-2$ & $140(74.1)$ & $46(57.5)$ & 0.007 \\
\hline $3-5$ & $49(25.9)$ & $34(42.5)$ & \\
\hline
\end{tabular}

Abbreviations: AFU, alpha-l-fucosidase; CEA, carcinoembryonic antigen; CA19-9, cancer antigen 19-9; CRS, clinical risk score.

Table 3. Univariate and multivariate analyses of the factors influencing OS by Cox proportional hazard model

\begin{tabular}{|c|c|c|c|c|}
\hline & \multicolumn{2}{|l|}{ Univariate analysis } & \multicolumn{2}{|l|}{ Multivariate analysis } \\
\hline & $\mathrm{HR}(95 \% \mathrm{CI})$ & $\mathbf{P}$ value & $\mathrm{HR}(95 \% \mathrm{CI})$ & $\mathbf{P}$ value \\
\hline \multicolumn{5}{|l|}{ Age (year) } \\
\hline$\leq 60$ vs. $>60$ & $1.374(0.923-2.048)$ & 0.118 & & \\
\hline \multicolumn{5}{|l|}{ Gender } \\
\hline Female vs. Male & $1.224(0.799-1.876)$ & 0.353 & & \\
\hline \multicolumn{5}{|l|}{ Primary tumor site } \\
\hline Colon vs. Rectum & $1.837(1.235-2.732)$ & 0.003 & $2.174(1.448-3.364)$ & $<0.001$ \\
\hline \multicolumn{5}{|l|}{ T stage } \\
\hline $1-3$ vs. 4 & $1.096(0.746-1.611)$ & 0.639 & & \\
\hline \multicolumn{5}{|l|}{ N stage } \\
\hline 0 vs. $1-2$ & 1.955 (1.271-3.005) & 0.002 & $2.414(1.552-3.754)$ & $<0.001$ \\
\hline \multicolumn{5}{|l|}{ Histological } \\
\hline $\begin{array}{l}\text { Well/moderate vs. } \\
\text { Poor }\end{array}$ & $1.472(0.861-2.518)$ & 0.157 & & \\
\hline \multicolumn{5}{|l|}{$\begin{array}{l}\text { Liver metastases } \\
\text { tumor size }(\mathrm{cm})\end{array}$} \\
\hline$\leq 2.3$ vs. $>2.3$ & $1.476(0.987-2.207)$ & 0.058 & & \\
\hline \multicolumn{5}{|l|}{$\begin{array}{l}\text { Liver metastases } \\
\text { number }\end{array}$} \\
\hline Single vs. Multiple & $1.685(1.128-2.518)$ & 0.011 & $2.027(1.343-3.058)$ & 0.001 \\
\hline \multicolumn{5}{|l|}{$\begin{array}{l}\text { Hepatic resection } \\
\text { timing }\end{array}$} \\
\hline $\begin{array}{l}\text { Synchronous vs. } \\
\text { Metachronous }\end{array}$ & $1.297(0.871-1.930)$ & 0.200 & & \\
\hline \multicolumn{5}{|l|}{ CEA (ng/ml) } \\
\hline$\leq 5$ vs. $>5$ & $1.245(0.829-1.870)$ & 0.290 & & \\
\hline \multicolumn{5}{|l|}{ CA199 (U/ml) } \\
\hline$\leq 37$ vs. $>37$ & $1.051(0.659-1.678)$ & 0.834 & & \\
\hline \multicolumn{5}{|l|}{ AFU (U/L) } \\
\hline$\leq 30.8$ vs. $>30.8$ & $0.601(0.367-0.984)$ & 0.043 & $0.438(0.264-0.728)$ & 0.001 \\
\hline
\end{tabular}




\section{Discussion}

AFU was reported to be a lysosomal protein [25], which catalyses the hydrolytic cleavage of terminal alpha-L-fucose residues in glycoproteins and glycolipids [26]. Alpha-L-fucose-containing molecules are key aspects for the progression of tumours, including haematogenous metastasis [16], tumour invasion through extracellular matrices [27], and upregulation of Notch signalling[28, 29], with implications for the epithelial-to-mesenchymal transition (EMT) and activation of cancer stem cells. AFU has been previously studied for its diagnostic value in several cancers. For example, AFU has been proven to be a potential diagnostic marker for HCC $[19,21]$ and CRC [30]. Another study showed that AFU in combination with CD26 can form a molecular model for diagnosis, especially for non-disseminated CRC [31]. The combination of AFU and TCH can have better diagnostic accuracy in comparison with their individual detection ability, which is helpful for differential diagnosis between malignant and non-tuberculous benign ascites [32]. In our study, we found that increased preoperative AFU in peripheral blood can predict better survival in CRLM patients undergoing hepatic resection. In a previous study, it was demonstrated that AFU can remove a-L-fucose from oligosaccharide sites on metastatic cancer cells [33]. Therefore, based on the results of our study, we hypothesized that high AFU expression could decrease the expression of fucose-containing molecules on the surface of colorectal cancer cells, thereby significantly inhibiting tumour cell invasion. That could explain the results of our study regarding why higher AFU can predict a better outcome. It might be expected that the enzyme AFU, which is involved in the removal of fucose-containing glycoproteins and glycolipids, could play an important role in the maintenance of the fucose content of aberrantly fucosylated glycoconjugates.

In a number of studies related to AFU, researchers used different methods to determine its cut-off value [34, 35]. In our study, the cut-off value for AFU was identified using X-tile software. This tool is used to assess the biological relationships between a biomarker and clinical outcome and to determine the cut-off points based on marker expression level [36]. This statistical method has been shown to be effective in a number of studies [35,37].

In particular, we found that AFU can be an efficient prognostic predictor for CRS low (score: 0-2) patients, not CRS high (score: 3-5) patients. The CRS introduced by Fong et al has been used in predicting recurrence after hepatic resection for metastatic colorectal cancer patients undergoing resection of liver metastases [24]. In our study, we performed
Kaplan-Meier analysis on the survival of patients stratified by groups with low CRS risk and high CRS risk. In the subgroup with low CRS risk, higher AFU was associated with favourable OS. However, in subgroups with high CRS risk, the result was not significant. This may be because patients with low-risk CRS have longer survival, so the relationship between AFU and prognosis can be observed. Our results suggest that for patients with low recurrence risk, there are still factors to which we should pay attention. In clinical applications for patients with low CRS, treatment should depend on the level of AFU. If patients belong to the high-AFU group (>30.8), they can consider undergoing less strict follow-up. However, larger and more objective prospective studies are needed to guide clinical decision making.

The lysosomal enzyme AFU coding gene FUCA-1 has been widely studied [38]. In breast cancer, it has been proven that FUCA-1 has independent prognostic value. Low FUCA-1 expression correlated with a significantly shorter relapse-free survival (RFS) as well as OS, while FUCA-1 overexpression was associated with a relatively good outcome [39]. Furthermore, decreased expression of the FUCA-1 gene was also found in human colorectal carcinomas compared with normal counterparts. A gradual decrease in FUCA-1 expression was observed along with the progression of CRC from earlier to advanced stages [11]. In highly aggressive and metastatic human tumours, the FUCA-1 gene is downregulated, possibly because its inactivation disturbs the fucosylation of proteins involved in cell adhesion, migration and metastases. Yuan et al hypothesized that a decrease in fucose content might alter the biological behaviour of breast cancer cells and, in particular the interaction among tumour cells, the ECM and endothelial cells, yielding new information for the diagnosis and treatment of metastases [16]. Furthermore, the expression of the FUCA-1 gene has been reported to be directly controlled by p53 [40, 41]. Mutated p53 has been associated with lower expression of FUCA-1 in human thyroid cancer cell lines [41].

However, this study has some limitations. First, it was a retrospective single-institution study. Several diseases, such as hepatitis and cirrhosis, which can affect the value of AFU, were not screened. This may lead to a selection bias. Second, numerous studies have shown different cut-off values for AFU level, so this requires further validation. Finally, the short duration of follow-up time was insufficient to evaluate longer survival outcomes.

In conclusion, patients with low AFU have various disadvantages in clinical background that can adversely affect long-term outcomes after hepatic 
resection. Particularly, for patients with lower CRS, AFU can predict postoperative survival more accurately. Higher preoperative AFU may become a convincing marker to estimate better survival outcomes for CLOM patients undergoing hepatic resection.

\section{Abbreviations}

AFU: alpha-l-fucosidase; CLOM: colorectal cancer liver oligometastasis; CRS: clinical risk score; OS: overall survival; CRC: colorectal cancer; CRLM: colorectal cancer liver metastasis; ESMO: European Society for Medical Oncology; OMD: oligometastatic disease; HCC: hepatocellular carcinoma; CEA: carcinoembryonic antigen; CT: computed tomography; MRI: magnetic resonance imaging; CA199: carbohydrate antigen 19-9; EMT: epithelial-to-mesenchymal transition; HR: hazard ratio; $\mathrm{CI}$ : confidence interval.

\section{Acknowledgements}

We would like to acknowledge the patients who participated in this study. We also gratefully acknowledge the assistance of the colleagues at State Key Laboratory of Oncology in South China and the Department of Colorectal Surgery in Sun Yat-sen University Cancer Center. The authenticity of the data in this article has been validated by uploading the key raw data onto the Research Data Deposit public platform (www.researchdata.org.cn), with the approval number as RDDA2018000892.

\section{Competing Interests}

The authors have declared that no competing interest exists.

\section{References}

1. Bray F, Ferlay J, Soerjomataram I, et al. Global cancer statistics 2018: GLOBOCAN estimates of incidence and mortality worldwide for 36 cancers in 185 countries. CA Cancer J Clin. 2018.

2. Gallinger S, Biagi JJ, Fletcher GG, et al. Liver resection for colorectal cancer metastases. Curr Oncol. 2013;20(3): e255-e265.

3. Van Cutsem E, Cervantes A, Adam R, et al. ESMO consensus guidelines for the management of patients with metastatic colorectal cancer. Ann Oncol. 2016;27(8):1386-1422.

4. Engels B, Gevaert T, Everaert H, et al. Phase II study of helical tomotherapy in the multidisciplinary treatment of oligometastatic colorectal cancer. Radiat Oncol. 2012; 7:34.

5. Van den Begin R, Engels B, Gevaert T, et al. Impact of inadequate respiratory motion management in SBRT for oligometastatic colorectal cancer. Radiother Oncol. 2014;113(2):235-239.

6. Reyes DK, Pienta KJ. The biology and treatment of oligometastatic cancer. Oncotarget. 2015;6(11):8491-8524.

7. Weiser MR, Jarnagin WR, Saltz LB. Colorectal cancer patients with oligometastatic liver disease: what is the optimal approach? Oncology (Williston Park). 2013;27(11):1074-1078.

8. Intra J, Perotti ME, Pavesi G, et al. Comparative and phylogenetic analysis of alpha-L-fucosidase genes. Gene. 2007;392(1-2):34-46.

9. Krause A, Combaret V, Iacono I, et al. Genome-wide analysis of gene expression in neuroblastomas detected by mass screening. Cancer Lett. 2005;225(1):111-120

10. Daves MH, Hilsenbeck SG, Lau CC, et al. Meta-analysis of multiple microarray datasets reveals a common gene signature of metastasis in solid tumors. Bmc Med Genomics. 2011:4:56.
11. Otero-Estevez O, Martinez-Fernandez M, Vazquez-Iglesias L, et al. Decreased expression of alpha-L-fucosidase gene FUCA1 in human colorectal tumors. Int J Mol Sci. 2013;14(8):16986-16998.

12. Vajaria BN, Patel KR, Begum R, et al. Evaluation of serum and salivary total sialic acid and alpha-l-fucosidase in patients with oral precancerous conditions and oral cancer. Oral Surg Oral Med Oral Pathol Oral Radiol. 2013;115(6):764-771.

13. Fernandez-Rodriguez J, Paez DLCM, Martinez-Zorzano VS, et al. Fucose levels in sera and in tumours of colorectal adenocarcinoma patients. Cancer Lett. 1997;121(2):147-153.

14. Huang W, Li X. [Significance of fucose expression in lung carcinoma and their brain metastases]. Zhonghua Bing Li Xue Za Zhi. 2000;29(4):259-262.

15. Listinsky JJ, Listinsky CM, Alapati V, et al. Cell surface fucose ablation as a therapeutic strategy for malignant neoplasms. Adv Anat Pathol. 2001;8(6):330-337.

16. Yuan K, Kucik D, Singh RK, et al. Alterations in human breast cancer adhesion-motility in response to changes in cell surface glycoproteins displaying alpha-L-fucose moieties. Int J Oncol. 2008;32(4):797-807.

17. Conti F, Dall'Agata M, Gramenzi A, et al. Biomarkers for the early diagnosis of bacterial infection and the surveillance of hepatocellular carcinoma in cirrhosis. Biomark Med. 2015;9(12):1343-1351.

18. Deugnier $Y$, David V, Brissot $P$, et al. Serum alpha-L-fucosidase: a new marker for the diagnosis of primary hepatic carcinoma? Hepatology. 1984;4(5):889-892.

19. Giardina MG, Matarazzo M, Varriale A, et al. Serum alpha-L-fucosidase. A useful marker in the diagnosis of hepatocellular carcinoma. Cancer-Am Cancer Soc. 1992;70(5):1044-1048.

20. Hutchinson WL, Johnson PJ, Du MQ, et al. Serum and tissue alpha-L-fucosidase activity in the pre-clinical and clinical stages of hepatocellular carcinoma. Clin Sci (Lond). 1991;81(2):177-182.

21. Takahashi H, Saibara T, Iwamura S, et al. Serum alpha-L-fucosidase activity and tumor size in hepatocellular carcinoma. Hepatology. 1994;19(6):1414-1417.

22. Zhou Y, Ma X, Wu J, et al. [Preoperative serum alpha-1-fucosidase as an early-recurrent indicator for hepatocellular carcinoma following curative resection]. Zhonghua Yi Xue Za Zhi. 2014;94(46):3623-3628.

23. Ayude D, Páez De La Cadena M, Martínez-Zorzano VS, et al. Preoperative Serum Alpha- $L$-Fucosidase Activity as a Prognostic Marker in Colorectal Cancer. Oncology. 2002;64(1):36-45.

24. Fong Y, Fortner J, Sun RL, et al. Clinical score for predicting recurrence after hepatic resection for metastatic colorectal cancer: analysis of 1001 consecutive cases. Ann Surg. 1999;230(3):309-318, 318-321.

25. Sleat DE, Jadot $M$, Lobel P. Lysosomal proteomics and disease. Proteomics Clin Appl. 2007;1(9):1134-1146.

26. Listinsky JJ, Siegal GP, Listinsky CM. The emerging importance of alpha-L-fucose in human breast cancer: a review. Am J Transl Res. 2011;3(4):292-322.

27. Yuan K, Listinsky CM, Singh RK, et al. Cell surface associated alpha-L-fucose moieties modulate human breast cancer neoplastic progression. Pathol Oncol Res. 2008;14(2):145-156.

28. Ayukawa T, Matsumoto K, Ishikawa HO, et al. Rescue of Notch signaling in cells incapable of GDP-L-fucose synthesis by gap junction transfer of GDP-L-fucose in Drosophila. Proc Natl Acad Sci U S A. 2012;109(38):15318-15323.

29. Sturla L, Rampal R, Haltiwanger RS, et al. Differential terminal fucosylation of $\mathrm{N}$-linked glycans versus protein O-fucosylation in leukocyte adhesion deficiency type II (CDG IIc). J Biol Chem. 2003;278(29):26727-26733.

30. Delacadena M, Fernandez J, Decarlos A, et al. Low levels of alpha-L-fucosidase activity in colorectal cancer are due to decreased amounts of the enzymatic protein and are related with Dukes' stage. Int J Oncol. 1996;9(4):747-754.

31. Ayude D, Paez DLCM, Cordero OJ, et al. Clinical interest of the combined use of serum CD26 and alpha-L-fucosidase in the early diagnosis of colorectal cancer. Dis Markers. 2003;19(6):267-272.

32. Zhang H, Li F, Wei Q, et al. Value of combined detection of AFU and TCH in differential diagnosis between malignant and non-tuberculous benign ascites. Med Oncol. 2011;28 Suppl 1: S670-S674.

33. Yuan K, Listinsky CM, Singh RK, et al. Cell surface associated alpha-L-fucose moieties modulate human breast cancer neoplastic progression. Pathol Oncol Res. 2008;14(2):145-156

34. Shuang Z, Mao Y, Lin G, et al. Alpha-L-Fucosidase Serves as a Prognostic Indicator for Intrahepatic Cholangiocarcinoma and Inhibits Its Invasion Capacity. Biomed Res Int. 2018; 2018:1-7.

35. Wang K, Guo W, Li N, et al. Alpha-1-fucosidase as a prognostic indicator for hepatocellular carcinoma following hepatectomy: a large-scale, long-term study. Brit J Cancer. 2014;110(7):1811-1819.

36. Camp RL, Dolled-Filhart M, Rimm DL. X-tile: a new bio-informatics tool for biomarker assessment and outcome-based cut-point optimization. Clin Cancer Res. 2004;10(21):7252-7259.

37. Xiao J, Ye ZS, Wei SH, et al. Prognostic significance of pretreatment serum carcinoembryonic antigen levels in gastric cancer with pathological lymph node-negative: A large sample single-center retrospective study. World J Gastroenterol. 2017;23(48):8562-8569.

38. Willems PJ, Seo HC, Coucke P, et al. Spectrum of mutations in fucosidosis. Eur J Hum Genet. 1999;7(1):60-67. 
39. Milde-Langosch K, Karn T, Schmidt M, et al. Prognostic relevance of glycosylation-associated genes in breast cancer. Breast Cancer Res Treat. 2014;145(2):295-305.

40. Baudot $\mathrm{AD}$, Crighton $\mathrm{D}, \mathrm{O}$ 'Prey J, et al. p53 directly regulates the glycosidase FUCA1 to promote chemotherapy-induced cell death. Cell Cycle. 2016;15(17):2299-2308.

41. Tsuchida N, Ikeda MA, Iotashino U, et al. FUCA1 is induced by wild-type 553 and expressed at different levels in thyroid cancers depending on p53 status. Int J Oncol. 2017;50(6):2043-2048. 